\title{
Assessment of Dual Tree Complex Wavelet Transform to improve SNR in collaboration with Neuro-Fuzzy System for Heart Sound Identification
}

Bassam Al-Naami ( $\square$ b.naami@hu.edu.jo )

Hashemite University https://orcid.org/0000-0002-1249-009X

Hossam Fraihat

Al-Ahliyaa Amman University

Jamal Al-Nabulsi

Al-Ahliyya Amman University

Abdel-Razzak Al-Hinnawi

Isra University

\section{Research Article}

Keywords: Heart Sound Classification, Dual Tree Complex Wavelet Transform, Adaptive Neuro Fuzzy Inference System, De-noising signals

Posted Date: August 9th, 2021

DOl: https://doi.org/10.21203/rs.3.rs-628636/v1

License: (c) (i) This work is licensed under a Creative Commons Attribution 4.0 International License. Read Full License

Version of Record: A version of this preprint was published at Electronics on March 17th, 2022. See the published version at https://doi.org/10.3390/electronics11060938. 


\title{
Assessment of Dual Tree Complex Wavelet Transform to improve SNR in collaboration with Neuro-Fuzzy System for Heart Sound Identification
}

\author{
Bassam Al-Naami ${ }^{1,2}$, Hossam Fraihat ${ }^{3}$, Jamal Al-Nabulsi², and Abdel-Razzak \\ Al-Hinnawi ${ }^{4}$ \\ ${ }^{1}$ Department of Biomedical Engineering, Faculty of Engineering, The Hashemite \\ University, PO Box 330127, Zarqa 13133, Jordan \\ ${ }^{2}$ Department of Medical Engineering, Al-Ahliyya Amman University, Amman 19328, Jordan \\ ${ }^{3}$ Department of Electrical Engineering, Al-Ahliyya Amman University, Amman 19328, \\ Jordan \\ ${ }^{4}$ Faculty of Allied Medical Sciences, Al-Isra University, PO Box 33 and 22, Amman \\ 11622, Jordan \\ Corresponding author: Bassam Al-Naami (e-mail: b.naami@hu.edu.jo)
}

\begin{abstract}
Here we propose a novel de-noising method to improve the outcome of heart sound (HS)-based heart condition identification. We applied Dual Tree Complex Wavelet Transform (DTCWT) in collaboration with Adaptive Neuro Fuzzy Inference System (ANFIS) classifier. The method consisted of three steps. First, preprocess to eliminate $50 \mathrm{~Hz}$ noise. Second, application of DTCWT to de-noise and reconstruct time-domain HS signal. Third, evaluation of ANFIS on total 2735 HS recordings from an international dataset (PhysioNet Challenge 2016). The signal-to-noise ratio $(\mathrm{SNR})$ with DTCWT was significantly improved $(\mathrm{p}<0.001)$ as compared to original HS recordings. Quantitatively, there was a $11 \%$ increase in SNR after DTCWT, representing a significant improvement in de-noising HS. In addition, the ANFIS, using six time-domain features, resulted in $55-86 \%$ precision, $51-98 \%$ recall, $53-86 \%$ f-score, and 54-86\% MAcc in comparison to other attempts on the same dataset. Therefore, DTCWT is a successful technique in de-noising information such as HS recordings. The adaptive property of ANFIS exhibited capability in classifying HS recordings.
\end{abstract}

Keywords Heart Sound Classification. Dual Tree Complex Wavelet Transform. Adaptive Neuro Fuzzy Inference System. De-noising signals. 


\section{Introduction}

Healthy cardiac valves (CV) play essential roles in our overall health [1]. Functional conditions of $\mathrm{CV}$ can be examined by the sounds of $\mathrm{CV}$ opening and closing in heart beats. The heart sounds (HS) measured by phonocardiograph (PCG) are consisted of 4 different sounds, S1, S2, S3 and S4 [1, 3]. Various CV diseases can be detected from the heart sounds, mostly during S1 and S2 but the accuracy of such diagnosis is largely depending on experience and expertise of a cardiologist [2,3]. However, PCG is a complicated non-stationary signal in nonlinear low frequency, which can be easily interfered by surrounding signal sources [3, 4].

Since S1-S2 section is the vital portion of HS in diagnosis for CV diseases from PCG, human errors in the diagnosis may cause life hazard [3]. To aid cardiologist in distinguishing healthy Normal Heart Sounds (NrHS) from Pathological Heart Conditions (HC) various signal processing and artificial intelligence algorithm have been applied to S1 and S2 [2]. To date, there have been 1,347 studies for algorithms for automatic analysis and classification of HS [2]. The earliest attempt was in 1963 by Gerbarg et al. who applied merely signal processing analysis on 1000 HS recordings [1]. Since then, hundreds of attempts have been reported that applied the more advanced information technology, such as the artificial neural networks (ANN) classifiers and mathematical transforms including Fourier transform (FT) and wavelet transform (WT). Most of previously applied signaling processes had a three steps of construction: a de-noising process, the extraction of signal "features" to discern HC from NrHS, and application of a "classifier" such as ANN or Deep Learning (DL) (i.e. Machine learning) [5]. The signal features are usually extracted either from time domain, wavelet domain, frequency domain, or morphological operations [6-9]. Many types of ANN and DL techniques have been applied on different datasets with a range of accuracy [10-13].To facilitate the research to develop an efficient and reliable computer algorithm supporting the diagnosis of heart diseases, international databases have been established containing large PCG data sets of HC and NrHS. PhysioNet Challenge 2016 is an example, which was Massachusetts Institute of Technology (MIT) in the Unites States [14].

The Physio-Net Challenge 2016 dataset, as a single data source, has allowed quantitative comparisons in between different signal processing techniques and associated algorithm [4, 15-28]. A number of different techniques and algorithm have been tested using Physio-Net Challenge 2016 data set, including AdaBoost Classifier, Gram Polynomials and probabilistic Neural Networks, and Convolutional Neural Network (CNN), tensor technique, Ensemble feature-based Feed-Forward Neural Networks (FNN), Drop Connected Neural Networks (DCNN), LogitBoost, Random Forest, and Cost-Sensitive Classifier [4, 15, 16, 26-28]. The outcome indicated a range of classification accuracies from $75 \%$ to $92 \%$ from the dataset. We generated Table 4 , in the discussion section, to list some of the remarkable and relevant efforts.

In this study, we tested a new technique to de-noise the HS using the Dual Tree Complex Wavelet Transform (DTCWT) using the Physio-Net Challenge 2016 data set. The DTCWT was 
modified from discrete wavelet transform (DWT) to overcome the drawbacks during sampling procedure, leading to inadequate noise elimination primarily due to failure in the anti-aliasing and shifting invariance properties [29].

Our findings from 2,735 HS signals indicated that the signal-to-noise ratio (SNR) was improved by DTCWT. A set of time-domain HS signal features was extracted and analyzed using the Adaptive Neuro Fuzzy Inference System (ANFIS). Our study may suggest the efficiency and reliability of DTCWT in processing HS data, followed by the accurate diagnosis as compared to other studies on PhysioNet 2016.

\section{Materials and methods}

The PhysioNet Challenge is an international dataset of HS signals, acquired from multiple sites around the world including non-clinical and clinical health organizations, professional and nonprofessional supervision, and various medical recording instruments. It is consisted of five classes categorized as A, B, C, D, and E, containing NrHS and HC signals [14]. There is a total of 3,153 HS in the dataset, with unevenly distributed HC severity among classes [5]. In addition, each class was constructed to contain some emergency external noise such as uncontrolled environment voices $[5,14]$.

In this research, we downloaded the HS recordings from all the five classes in the dataset. These HS recordings were divided into training and test sets using the $80-20 \%$ split protocol, as widely applied in ANN. We combined the class $C$ data with class D due to their low numbers of HS signals to allow $80-20 \%$ ANN split protocol. To meet the objectives of this paper, we discarded obvious distorted HS recordings that contained unusual external sounds using our experience as biomedical engineers, so we had 2735 out of $3,153 \mathrm{HS}$ recordings, approximately $87 \%$ of the entire dataset, as illustrated in Table 1. As the HS signal is periodic where S1 and S2 are repetitive, we assigned 5 seconds $\mathrm{HS}$ duration to cover the $\mathrm{S} 1$ and $\mathrm{S} 2$ and sampled the signals at 2,000 $\mathrm{Hz}$, as per the specifications of the PhysioNet.

Table1 Dataset Distribution

\begin{tabular}{|c|c|c|c|c|}
\hline Class & SETS & \# Subjects & Abnormal HC & Normal HS \\
\hline \multirow[t]{2}{*}{$\mathrm{A}$} & Training & 313 & 218 & 95 \\
\hline & Test & 78 & 54 & 24 \\
\hline \multirow[t]{2}{*}{$\mathrm{B}$} & Training & 356 & 72 & 284 \\
\hline & Test & 88 & 18 & 70 \\
\hline \multirow[t]{2}{*}{$\mathrm{C}+\mathrm{D}$} & Training & 50 & 28 & 22 \\
\hline & Test & 12 & 7 & 5 \\
\hline \multirow[t]{2}{*}{$\mathrm{E}$} & Training & 1472 & 121 & 1351 \\
\hline & Test & 366 & 28 & 338 \\
\hline \multirow[t]{2}{*}{$\mathrm{A}, \mathrm{B}, \mathrm{C}, \mathrm{D}, \mathrm{E}$} & Training & 2191 & 439 & 1752 \\
\hline & Test & 544 & 107 & 437 \\
\hline Total HS Signals & & & & \\
\hline
\end{tabular}

Figure 1 shows the block diagram of the proposed method. It differs from our previous work [4] in which we investigated a new de-noising technique (DTCWT) and employed ANFIS 
using time domain features instead of spectral domain. To best of our knowledge, the DTCWT, which is a tool that permits quantitative SNR analysis, has not been explored for HS recordings in PhysioNet Challenge 2016.

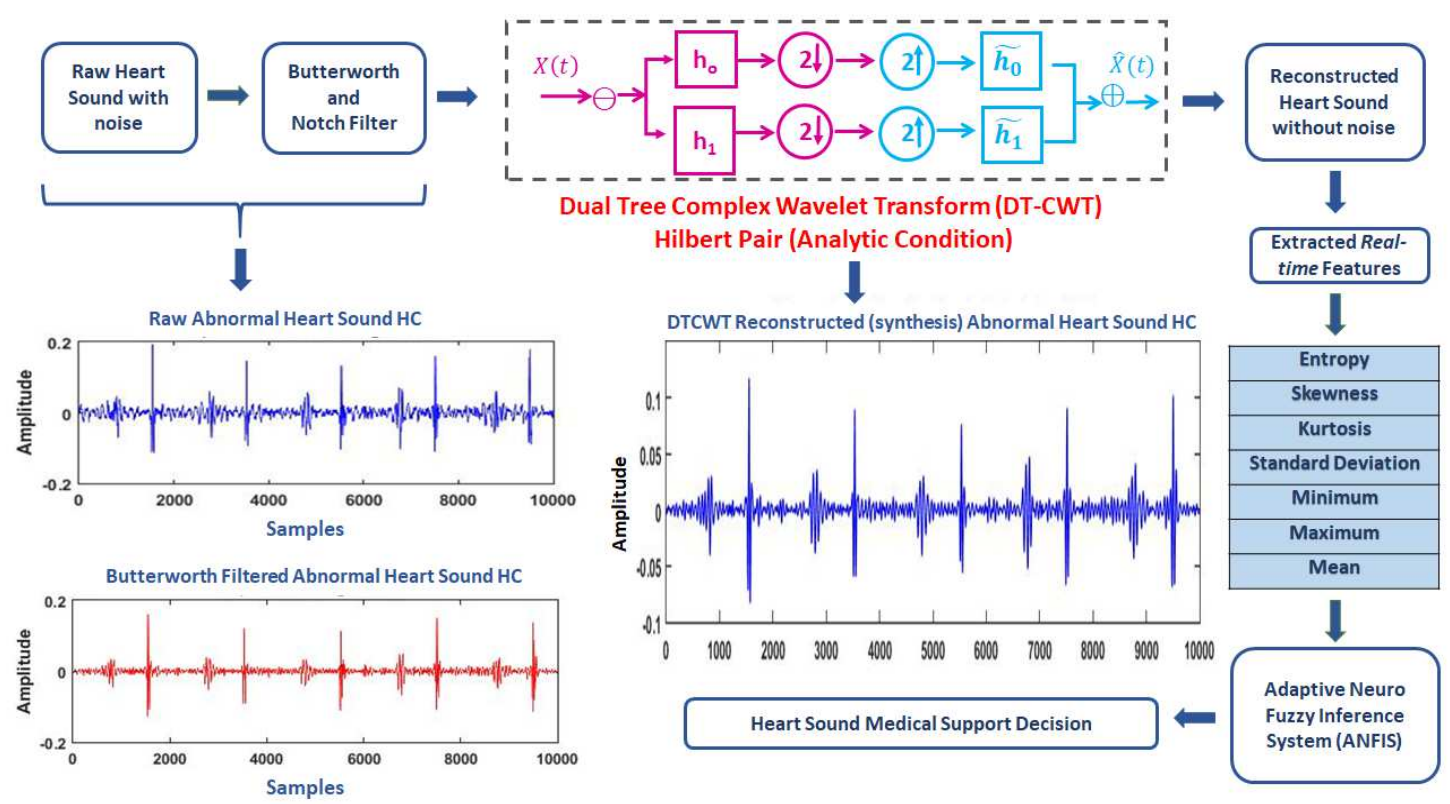

FIG. 1 The Block diagram of the proposed HS recordings de-noising and classifier

\section{Pre-processing of HS Signal}

Each HS signal was pre-processed by applying a Notch filter to eliminate the primary $50-60 \mathrm{~Hz}$ noise. Then, a Butterworth filter was applied using two cutoff frequencies; $F C 1=0.025$ and $\mathrm{FC} 2=0.4[30,31]$. Figure 1 shows examples of on an $\mathrm{HC}$ after applying previous steps.

\section{Dual Tree Complex Wavelet Transform}

The DWT, developed to aid signal analysis, suffers filtering difficulties during sampling procedure, leading to inadequate noise elimination due to failure in the anti-aliasing and shifting invariance properties [29]. To overcome such limitations, DTCWT was developed to enhance noise abolition by DWT [31]. The DTCWT includes several sequential steps during the decomposition and reconstruction process. As these two processes are applied to the real and imaginary constituents of the signal so it is denoted as Dual Tree. Each tree is a one-dimensional DWT presentation consisted of the real and imaginary parts, which is referred as Complex Wavelet Transform. As shown in Figure 2, each tree can be considered as a Filter-Bank (FB) tree containing low pass filter $\left(\mathrm{h}_{0}\right.$ or $\left.\mathrm{g}_{0}\right)$ and high pass filter $\left(h_{1}\right.$ and $\left.g_{1}\right)$. During the decompose process, the low and high pass filters divide signals into Approximate Coefficients (cA) and Detail Coefficients (cD). The size of $\mathrm{cA}$ and $\mathrm{cD}$ are successively decreased by factor of 2 at each decomposition level. The factor of 2 was selected because it is the optimum DTCWT performance as indicated in ref [29]. During the reconstruction process, a reverse procedure is applied to rebuild the signal successively with progressive de-noising, leading to optimal reconstruction with the minimum anti-aliasing and shift invariance impacts [29]. 
Particularly, the DTCWT satisfies the well-known condition of Hilbert Analytic, which is illustrated in top of Figure 1 (denoted in dashed box), indicating that the scaling complex function and the wavelet complex function collaborate to generate Hilbert pair. Equation 1 shows its mathematical presentation as previously described $[29,31,32]$.

$$
\psi_{c}(t)=\psi_{r}(t)+j \psi_{i}(t)
$$

Where $\psi_{r}(t)$ is real (even), $j \psi_{i}(t)$ is imaginary (odd), and $\psi_{c}(t)$ is the DTCWT signal (analytic). The best performing DTCWT design can be achieved $[31,33]$ when internal structure of FBs satisfies the following three requirements:

1. Perfect reconstruction (PR) to make the reconstructed signal $\hat{X}(t)$ identical to the original (input) signal.

2. Successful application of half sample shift of low pass filters (ho and go).

3. The shifting procedure extended to one sample in the first divergence in the dual tree with respect to go and g1, respectively.

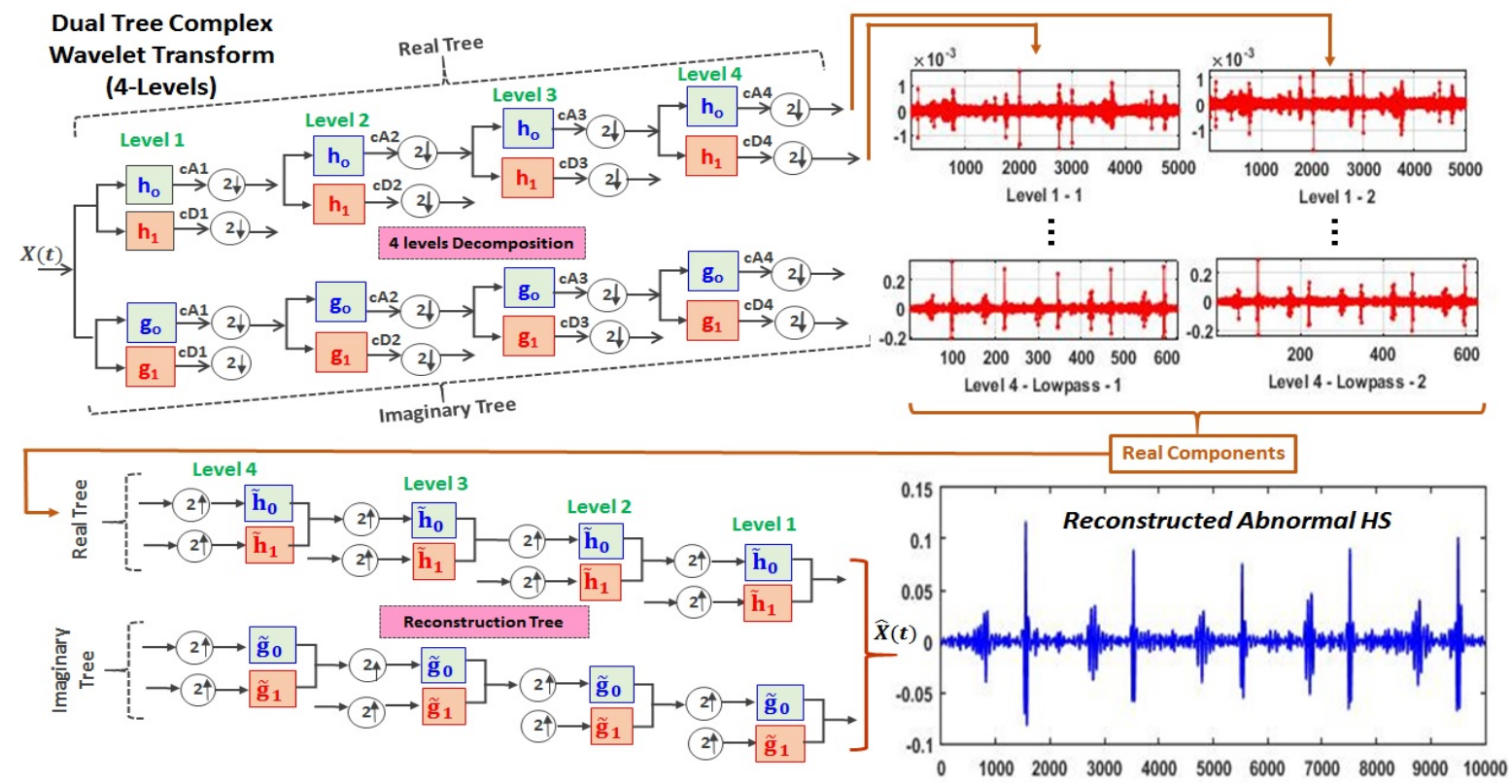

FIG. 2 One Dimensional DTCWT for analysis FB (decompose) and synthesis FB (reconstruct). X(t) represents the input signal. cA1, cA2, cA3 and cA4 denote the Approximate Coefficients at level 1, level 2, level3, and level 4, respectively, while cD1, cD2, cD3, and cD4 denote the Detail Coefficients at level 1, level 2, level3, and level 4, respectively. $\hat{X}(t)$ is the reconstructed output signal [2].

Therefore, DTCWT solves drawbacks in DWT by permitting q-shift and anti-aliasing during analysis and synthesis processes, according to Hilbert pair. This suggests that DTCWT may lead to better de-noising performance. Subsequently, the SNR of HS signals is expected to improve, consequently allowing more precise time-domain feature extraction. 


\section{SNR Calculations}

HS signals frequently contain many types of ambient noises, such as electronic circuit noise and interface between electrodes and skin [5]. It is often that a type "A" noise is blocked by another noise in type "B" that makes it challenging to precisely assign types of the embedded noises in the HS signals $[34,35]$. Therefore, it is recommended for the SNR to be calculated depending on the value of accumulative residual noises, and we calculated the SNR of HS using equation (2):

$$
\text { SNR }=\text { mean }\left(\text { Noisy_HS }{ }^{2}\right) / \text { mean }\left(\text { Residual_Noise }{ }^{2}\right)
$$

Where,

$$
\text { Residual_Noise }=\text { Noisy_HS }- \text { Denoised_HS } .
$$

It was converted to decibels (dB) by using equation (3).

$$
\mathrm{SNR}_{\mathrm{dB}}=10 * \log _{10}(\mathrm{SNR})
$$

Then, the SNR percentage difference (SNR\%) after DTCWT was calculated by equation (4):

$$
\text { SNR } \%=\frac{\text { Mean SNR after DTCWT }- \text { Mean SNR before DTCWT }}{\text { Mean SNR before DTCWT }}
$$

\section{Feature Extractions}

From HS signals after DTCW, we extracted a set of seven features, including Entropy, Skewness, Kurtosis, Standard Deviation (STDev), Minimum (min), Maximum (max), and Mean as listed in topright of Figure 1. These features were calculated in the training NrHS and HC signals after applying the DTCWT. Then, statistical significance between HC and NrHS measurements of the extracted features was assessed. The results indicated that all the features had yielded significant difference between NrHS and HC $(p<0.05)$, excepting for the mean.

The six features showing significant improvements, including Entropy, Skewness, Kurtosis, STDev, Max, and Min, were normalized between 0 and 1 prior to be applied for the ANFIS using the equation (5) adopted from a previous study [4]:

$$
\overrightarrow{\mathrm{F}}_{\mathrm{j}, \text { normalized }}=\left(\overrightarrow{\mathrm{F}}_{\mathrm{j}}-\mathrm{F}_{\mathrm{j}, \text { min }}\right) /\left(\mathrm{F}_{\mathrm{j}, \text { max }}-\mathrm{F}_{\mathrm{j}, \text { min }}\right)
$$

Where, $F_{j}$ and $F_{j, n o r m a l i z e d}$ are the original and normalized j-th feature, respectively; $F_{j, \min }$ and $F_{j, \max }$ are the minimum and the maximum of the $j$-th feature values calculated for all 2,735 samples, respectively. In other words, the $j$-th feature ( $\mathrm{j}=1$ to 6 ) for $\mathrm{n}$ samples ( $\mathrm{n}=1$ to 2735 ) was normalized 
between 0 and 1 values. Thus, the classification process, described in the following section, should not affected by different magnitudes of HS signal.

\section{Adaptive Neuro Fuzzy Inference System (ANFIS)}

The ANFIS is a machine-learning (ML)-based classifier algorithm that have been used for a variety of engineering problems in biomedical fields. It is a rule-based method originally developed by Jang [36]. The ANFIS has the ability of ANN ML that exploits fuzzy inference system to deduce decisions by a fuzzy logic method that takes in the account the membership degree of input-output variables [36]. The ANFIS architecture has two fuzzy if-then rules based on the Sugeno model. It has two set of input rules, which are applied to generate one output. The connection between the two input rules and the Sugeno fuzzy output is reconstructed in five layers of nodes, two layers are adaptive with flexibility, while the other three are fixed.

We applied ANFIS to each class of the HS dataset. Figure 3 shows an example of the ANFIS outputs on the test set in class A. The blue color indicates HC signals, while red color indicates NrHS signals. The ANFIS output was set to either 1 if pathology is predicted, or 2 if pathology is not predicted. Finally, the ANFIS performance was analyzed by the PRECISION and RECALL statistical metrics using Equations 6, 7, and 8, as in the following:

$$
\begin{gathered}
\text { Precision }=\mathrm{TP} /(\mathrm{TP}+\mathrm{FP}) \quad(6) \\
\text { RECALL }=\mathrm{TP} /(\mathrm{TP}+\mathrm{FN}) \\
\text { F-score }=2 * \text { precision*RECALL } /[\text { precision }+ \text { RECALL }]
\end{gathered}
$$

Where:

TP: true positive represents the HC-pathological samples detected correctly

FP: false positive represents the NrHS-normal samples detected as abnormal

TN: true negative represents the NrHS-normal samples detected correctly

FN: false negative represents the $\mathrm{HC}$ samples detected as $\mathrm{NrHS}$

Additionally, the modified accuracy (MAcc) was calculated, which is the average of precision and recall rates (i.e. (precision+ recall)/2). 


\section{ANFIS Testing Results on Calss A}

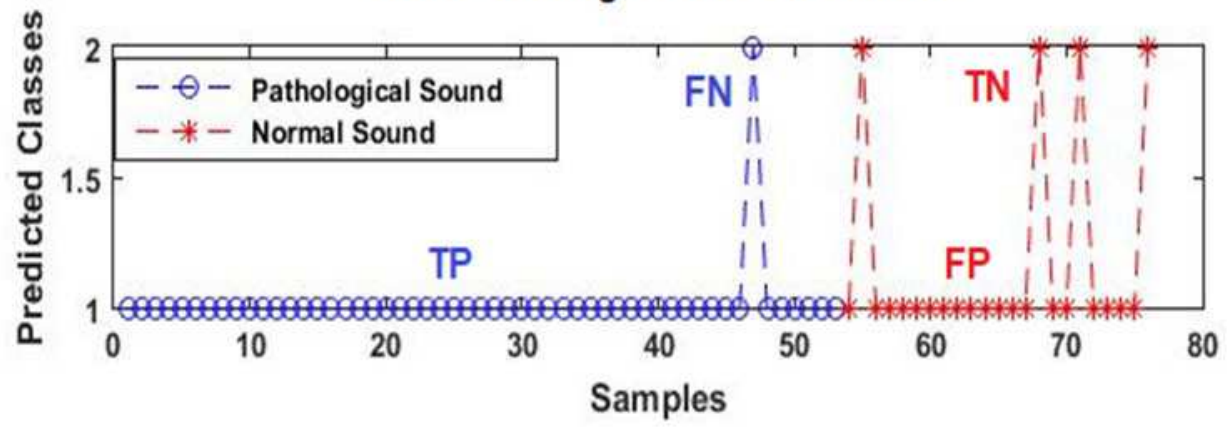

FIG. 3 ANFIS testing results for class A.

\section{Results}

For each HS signal (Normal or abnormal), the SNR was calculated twice before and after applying DTCWT. Table 2 shows the Average (AVG) and Standard Deviation (STDev) of SNR measurements on each class in the data set. It also shows the SNR percentage difference calculated from the equation 4 and statistical difference by z-test between SNR measurements. The AVG and STDev values indicates that SNR was increased after applying DTCWT with statistical significance (p-value $<0.001$ ).

Table2 The SNR measurements before and after DTCWT for all HS recordings in the dataset

\begin{tabular}{|c|c|c|c|c|c|}
\hline \multicolumn{2}{|c|}{$\begin{array}{l}\text { Dataset } \\
\text { (Before and after DTCWT) }\end{array}$} & $\begin{array}{l}\text { Average } \text { SNR } \\
{[\mathrm{dB}]}\end{array}$ & $\begin{array}{l}\text { SNR Standard } \\
\text { Deviation }[\mathrm{dB}]\end{array}$ & $\begin{array}{l}\text { SNR Percentage } \\
\text { Difference }[\mathrm{dB}]\end{array}$ & $\begin{array}{l}\text { Statistical } \\
\text { Significance } \\
\text { P-value }\end{array}$ \\
\hline \multirow{2}{*}{ Class A } & Before & 7.1 & 2.4 & \multirow{2}{*}{+0.11} & \multirow{2}{*}{$<0.001$} \\
\hline & After & 7.9 & 2.4 & & \\
\hline \multirow{2}{*}{ Class B } & Before & 0.7 & 0.6 & \multirow{2}{*}{+13.96} & \multirow{2}{*}{$<0.001$} \\
\hline & After & 10.8 & 3.5 & & \\
\hline \multirow{2}{*}{ Class C $+\mathrm{D}$} & Before & 5.6 & 3.7 & \multirow{2}{*}{+0.58} & \multirow{2}{*}{$<0.001$} \\
\hline & After & 8.9 & 4.0 & & \\
\hline \multirow{2}{*}{ Class E } & Before & 5.6 & 3.0 & \multirow{2}{*}{+0.65} & \multirow{2}{*}{$<0.001$} \\
\hline & After & 9.3 & 4.8 & & \\
\hline
\end{tabular}

In the second experiment, we calculated the time-domain features for each HS signal after DTCWT. Then the ANFIS classifier was trained and applied to each test set. The rate of recall, precision, and F-score was reported on each class in the dataset. The MAcc, which is often used in recent literature, was also reported. Table 3 shows that the average precision, recall, F-score, and MAcc were $0.68,0.81,0.74,75 \%$, respectively. Whilst, Figure 4 shows the box-plot of SNR measurements on HS recordings for the five classes in dataset (Table 1), indicating the increment in SNR after 
applying DTCWT. Finally, we compared our findings with several previous findings with wavelet transform and other approaches applied on PhysioNet challenge 2016 (Table 4).

Table 3 ANFIS Performance on dataset

\begin{tabular}{lllll}
\hline \hline Data set & Precision & Recall & F-Score & MAcc [\%] \\
\hline Class A & 0.73 & 0.98 & 0.84 & 85.5 \\
Class C+D & 0.86 & 0.87 & 0.86 & 86.0 \\
Class B & 0.55 & 0.89 & 0.68 & 72.0 \\
Class E & 0.56 & 0.51 & 0.53 & 53.5 \\
Average (A,B,C,D,E) & 0.68 & 0.81 & 0.74 & 74.5 \\
\hline
\end{tabular}

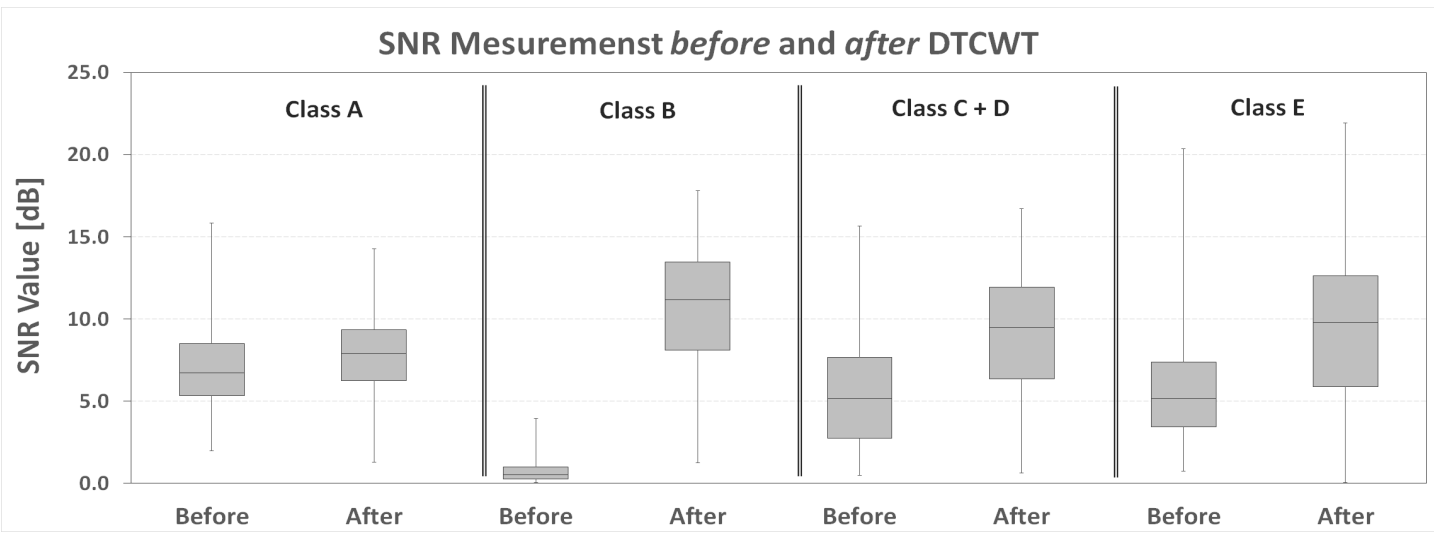

FIG. 4 SNR measurements on all HS recordings on all classes before and after utilizing DTCWT

\section{Discussion}

The results in Table 2 and Figure 4 demonstrate an improvement in the average SNR values after applying DTCWT in all classes with statistical significance $(\mathrm{p}<0.001)$, indicating the capability of DTCWT in de-noise HS recordings in PhysioNet 2016. Additionally, these results reveal inequality in the percentage of SNR improvement among the five different classes in the dataset. This may suggest that there were incoherent noise conditions during HS recordings likely due to various instruments, diverse recording conditions, and/or the potential human interferences. Therefore, the results, on one hand, represent numerical proofs that the different classes contain unequal noise conditions making some HS recordings impracticable or incorrect HS recordings. On the other hand, it shows that DTCWT can perform differently for different types and levels of embedded noises.

Furthermore, Table 2 shows that class A has the lowest SNR percentage difference with no change in STDev after DTCWT, whereas classes B and E exhibit the largest SNR difference along with large change in STDev after DTCWT. It suggests that class A exhibits the lowest level of 
embedded noises in the signal in comparison with the other classes. Similarly, the combined C+D, despite the smallest class sizes, resulted in a nearly equal STDev, suggesting the possible preservation of HS signal while elimination of noise through DTCWT. In contrast, the STDev was increased a number of decibels folds for classes B and E, likely indicating a possible weakening of HS signals. These findings are consistent with some studies suggesting that classes B and E contain the poorest quality of HS recordings [14, 21]. Kay E. et al. [16] argued that Class E should be excluded from PhysioNet Challenge 2016 as it contains clinically not practical HS recordings, which was also claimed by Gjoreski et al [5].

Whilst, analyzing the ANFIS classifying performances in Table 3 shows that ANFIS outputs on classes B and E appeared to be unsatisfactory in terms of accuracy. The ANFIS classifier is a machine code that can be trained to provide an optimal performance, so it is unlikely to be the reason. Therefore, the shortage in performance can be attributed to the quality of HS recordings, likely suggesting that classes B and E contained impractical HS signals, as claimed by references $[10,16$, $25,26]$. The potential solutions for this issue could be either to increase the number of input features to ANFIS classifier as in references [19, 21, 28, 37], or to segment S1 and S2 portions from the five seconds HS recordings as in references [17, 18, 20, 28].

Nonetheless, our ANFIS performed the mathematical calculations to achieve 73-86\% precision, $87-98 \%$ recall, $84-86 \%$ f-score, and approximately $86 \%$ MAcc (Table 3 ). This was achieved on all HS signals in classes A, C, and D, which presumably contained the correct S1-S2 segments, suggesting the adaptive property ANFIS with only six time-domain features performs well in classifying biosignals such as HS recordings. Our outcome is different from the previous finding by us [4] in many aspects. First, here we tested DTCWT for de-noise HS signals instead of Fourier bispectrum. Second, we employed time domain instead of frequency domain features as inputs to ANFIS. Third, we employed the kurtosis and skewness as inputs to ANFIS. Since we applied the new method in Figure 1 on 2735 instead of 1738 signals, we consider our outcomes in this paper to be more reliable. Therefore, combining the results in this paper along with our previous study [4] rationalized saying that the adaptive property in ANFIS with either time-domain or frequency-domain features performs well in classifying biosignals such as HS recordings.

When compared with previous attempts (Table 4) to apply various wavelet transform on PhysioNet Challenge 2016 dataset, we found the DTCWT is a feasible method to de-noise HS signals that has not been tested in literature before. Some of previous studies excluded doubtful HS signals in designing satisfactory HS decision support systems [4, 16, 20, 24, 31, 39]. However, there were other attempts which researchers included almost the entire 3,153 PhysioNet dataset so had to implement a high number of input features more than a hundred [25, 26, 37, 39]. All these attempts yielded a range of MAcc values from $75 \%$ to $86 \%$, which comes close to results in this paper, but we evaluated the DTCWT and ANFIS classifier. Although our accuracy is lower than some of the previous outcome, it is within the range previous reports when impractical HS recordings were excluded, particularly 
classes $\mathrm{B}$ and $\mathrm{E}$ as argued by references [10, 16, 24]. Finally, it is important to mention that this paper, in comparison to literature, quantitatively presented the SNR percentage difference after applying denoise, prevailing that DTCWT can perform differently for different types and levels of embedded noises in PhysioNet 2016.

It is noteworthy that the performance of DTCWT and ANFIS may be affected by several parameters. One of which is the Butterworth Filter cut-off frequencies. However, we speculate that any marginal change in the cut-off frequency would only make marginal change in performance [30]. The other parameters likely affecting the performance are the $\mathrm{cA}$ and $\mathrm{cD}$, the amplitude and detail coefficients in the DTCWT, respectively [29]. A follow-up prospective research of those two parameters may further improve the DTCWT reconstruction of HS signal, followed by an improvement of ANFIS performance. At the end, the number of decomposition and reconstruction levels in the DTCWT in Figure 2 also could have an impact.

\section{Conclusion}

In summary, this paper evaluated de-noising HS recordings by DTCWT, which has not been attempted. The results showed statistical significance in SNR improvement with substantial improvement, number of folds of [dB], in SNR percentage difference. The adaptive property of ANFIS classifier with 6-time domain features successfully resulted in an encouraging accuracy (74.5\%) on 2735 samples. If non-clinically-accepted HS recordings were excluded as in reports [10, 16,17, 20, 24 , $38,40]$, the proposed approach would result in $86 \%$ accuracy which come in range of accuracies in these reports, indicating the capability of DTCWT and ANFIS as a suggestive successful tool.

\section{Declarations}

Conflict of interest. The authors declare that they have no conflict of interest. 
Table 4 Literature Samples on Physio-Net Challenge 2016 Using Wavelet Transform

\begin{tabular}{|c|c|c|c|c|c|}
\hline Author & Methodology & $\begin{array}{l}\text { \# } \\
\text { Features }\end{array}$ & $\begin{array}{l}\text { Neural Network } \\
\text { Classifier }\end{array}$ & \# HS Samples & $\begin{array}{l}\text { Accuracy } \\
\text { MAcc } \%\end{array}$ \\
\hline $\begin{array}{l}\text { C. Potes et } \\
\text { al. [26] }\end{array}$ & $\begin{array}{l}\text { Time and } \\
\text { frequency- } \\
\text { domain } \\
\text { Features }\end{array}$ & 124 & $\mathrm{CNN}$ & 3240 & $86 \%$ \\
\hline $\begin{array}{l}\text { Whitaker, B. } \\
\text { M. et al. } \\
{[20]}\end{array}$ & $\begin{array}{l}\text { Sparse } \\
\text { coding }\end{array}$ & 20 & SVM & $2,153 *$ & $86.5 \%$ \\
\hline $\begin{array}{l}\text { M. } \\
\text { Tschannen } \\
\text { et al. [24] }\end{array}$ & $\begin{array}{l}\text { Wavelet deep } \\
\text { convolutional } \\
\text { neural } \\
\text { network } \\
(\mathrm{CNN})\end{array}$ & 25 & SVM & $1277 *$ & $81.2 \%$ \\
\hline $\begin{array}{l}\text { Goda et al. } \\
\text { [37] }\end{array}$ & $\begin{array}{l}\text { Wavelet } \\
\text { envelope } \\
\text { features }\end{array}$ & 128 & SVM & 3000 & $81.2 \%$ \\
\hline $\begin{array}{l}\text { Langley et } \\
\text { al. [38] }\end{array}$ & $\begin{array}{l}\text { Wavelet } \\
\text { Entropy }\end{array}$ & $\begin{array}{l}\text { wavelet } \\
\text { entropy }\end{array}$ & $\begin{array}{l}\text { Classification } \\
\text { algorithm }\end{array}$ & $400 *$ & $77 \%$ \\
\hline $\begin{array}{l}\text { Kay E. et al. } \\
{[16]}\end{array}$ & $\begin{array}{l}\text { Hidden semi- } \\
\text { Markov } \\
\text { model }\end{array}$ & 50 & $\begin{array}{l}\text { fully-connected, } \\
\text { two-hidden- } \\
\text { layer neural } \\
\text { network trained } \\
\text { by error } \\
\text { backpropagation }\end{array}$ & $\begin{array}{l}\text { All dataset } \\
\text { excluding Class E } \\
*\end{array}$ & $74.8 \%$ \\
\hline $\begin{array}{l}\text { Grzegorczyk } \\
\text { et al. [10] }\end{array}$ & $\begin{array}{l}\text { Algorithm } \\
\text { based on } \\
\text { Hidden } \\
\text { Markov } \\
\text { Model. }\end{array}$ & 48 & Neural networks & 3000 & $79 \%$ \\
\hline $\begin{array}{l}\text { Nilanon et } \\
\text { al. [25] }\end{array}$ & Spectrogram & $\begin{array}{l}\text { Many } \\
\text { time } \\
\text { windows }\end{array}$ & $\begin{array}{l}\text { SVM, CNN, } \\
\text { and Logistic } \\
\text { Regression (LR) }\end{array}$ & About 3000 & $68-80 \%$ \\
\hline $\begin{array}{l}\text { M. N. } \\
\text { Homsi et al. } \\
{[39]}\end{array}$ & $\begin{array}{l}\text { Nested } \\
\text { ensemble of } \\
\text { algorithms }\end{array}$ & 131 & $\begin{array}{l}\text { Random Forest, } \\
\text { LogitBoost and } \\
\text { a Cost-Sensitive } \\
\text { Classifier }\end{array}$ & $764 *$ & $84.48 \%$ \\
\hline This work & DTCWT & 6 & ANFIS & $\begin{array}{l}2735 \\
\text { dataset excluding } \\
\text { Classes B, E }\end{array}$ & $\begin{array}{l}74.5 \% \\
86 \%\end{array}$ \\
\hline
\end{tabular}

* Part of the 3153 HS recordings in the PhysioNet Challenge dataset were excluded. 


\section{References}

1. Gerbarg DS, Taranta A, Spagnuolo M, Hofler JJ (1963) Computer analysis of phonocardiograms. Prog Cardiovasc Dis 5:393-405. https://doi.org/10.1016/S0033-0620(63)80007-9

2. Dwivedi AK, Imtiaz SA, Rodriguez-Villegas E (2019) Algorithms for automatic analysis and classification of heart sounds-A systematic review. IEEE Access 7:8316-8345. https://doi.org/10.1109/ACCESS.2018.2889437

3. Shub C (2003) How to evaluate systolic murmurs. Can Fam Physician

4. Al-Naami B, Fraihat H, Gharaibeh N, Al-hinnawi AM (2020) A Framework Classification of Heart Sound Signals in PhysioNet Challenge 2016 Using High Order Statistics and Adaptive Neuro-Fuzzy Inference System. IEEE Access. https://doi.org/10.1109/ACCESS.2020.3043290

5. Gjoreski M, Gradisek A, Budna B, et al (2020) Machine Learning and End-to-End Deep Learning for the Detection of Chronic Heart Failure from Heart Sounds. IEEE Access 8:20313-20324. https://doi.org/10.1109/ACCESS.2020.2968900

6. De Vos JP, Blanckenberg MM (2007) Automated pediatric cardiac auscultation. IEEE Trans Biomed Eng 54:. https://doi.org/10.1109/TBME.2006.886660

7. Herzig J, Bickel A, Eitan A, Intrator N (2015) Monitoring Cardiac Stress Using Features Extracted From S1 Heart Sounds. IEEE Trans Biomed Eng 62:11691178. https://doi.org/10.1109/TBME.2014.2377695

8. Li H, Ren Y, Zhang G, et al (2019) Detection and Classification of Abnormities of First Heart Sound Using Empirical Wavelet Transform. IEEE Access 7:. https://doi.org/10.1109/ACCESS.2019.2943705

9. Schmidt SE, Graebe M, Toft E, Struijk JJ (2011) No evidence of nonlinear or chaotic behavior of cardiovascular murmurs. Biomed Signal Process Control. https://doi.org/10.1016/j.bspc.2010.07.003

10. Grzegorczyk I, Solinski M, Lepek M, et al (2016) PCG classification using a neural network approach. In: Computing in Cardiology. IEEE Computer Society, pp $1129-1132$

11. Chauhan S, Wang P, Sing Lim C, Anantharaman V (2008) A computer-aided MFCC-based HMM system for automatic auscultation. Comput Biol Med. https://doi.org/10.1016/j.compbiomed.2007.10.006

12. Saraçoglu R (2012) Hidden Markov model-based classification of heart valve disease with PCA for dimension reduction. Eng Appl Artif Intell 25:. https://doi.org/10.1016/j.engappai.2012.07.005

13. Cheng X, Huang J, Li Y, Gui G (2019) Design and application of a laconic heart sound neural network. IEEE Access https://doi.org/10.1109/ACCESS.2019.2934827

14. Liu C, Springer D, Li Q, et al (2016) An open access database for the evaluation of heart sound algorithms. Physiol Meas 37:2181-2213. https://doi.org/10.1088/0967-3334/37/12/2181

15. Beritelli F, Capizzi G, Lo Sciuto G, et al (2018) Automatic heart activity diagnosis based on Gram polynomials and probabilistic neural networks. Biomed Eng Lett 8:. https://doi.org/10.1007/s13534-017-0046-z

16. Kay E, Agarwal A (2017) DropConnected neural networks trained on timefrequency and inter-beat features for classifying heart sounds. Physiol Meas 38:1645-1657. https://doi.org/10.1088/1361-6579/aa6a3d

17. Liu C, Springer D, Clifford GD (2017) Performance of an open-source heart sound segmentation algorithm on eight independent databases. Physiol Meas 38:1730- 
1745. https://doi.org/10.1088/1361-6579/aa6e9f

18. Nabhan Homsi M, Warrick P (2017) Ensemble methods with outliers for phonocardiogram classification. Physiol Meas 38:. https://doi.org/10.1088/13616579/aa7982

19. Rubin J, Abreu R, Ganguli A, et al (2016) Classifying heart sound recordings using deep convolutional neural networks and mel-frequency cepstral coefficients. In: Computing in Cardiology

20. Whitaker BM, Suresha PB, Liu C, et al (2017) Combining sparse coding and timedomain features for heart sound classification. Physiol Meas 38:. https://doi.org/10.1088/1361-6579/aa7623

21. Plesinger F, Viscor I, Halamek J, et al (2017) Heart sounds analysis using probability assessment. Physiol Meas 38:. https://doi.org/10.1088/13616579/aa7620

22. Vernekar S, Nair S, Vijaysenan D, Ranjan R (2016) A novel approach for classification of normal/abnormal phonocardiogram recordings using temporal signal analysis and machine learning. In: Computing in Cardiology. IEEE Computer Society, pp 1141-1144

23. Maknickas V, Maknickas A (2017) Recognition of normal-abnormal phonocardiographic signals using deep convolutional neural networks and melfrequency spectral coefficients. Physiol Meas 38:. https://doi.org/10.1088/13616579/aa7841

24. Tschannen M, Kramer T, Marti G, et al (2016) Heart sound classification using deep structured features. In: Computing in Cardiology

25. Nilanon T, Yao J, Hao J, et al (2016) Normal / abnormal heart sound recordings classification using convolutional neural network. In: Computing in Cardiology

26. Potes C, Parvaneh S, Rahman A, Conroy B (2016) Ensemble of feature-based and deep learning-based classifiers for detection of abnormal heart sounds. In: Computing in Cardiology

27. Diaz Bobillo IJ (2016) A tensor approach to heart sound classification. In: Computing in Cardiology

28. Zabihi M, Rad AB, Kiranyaz S, et al (2016) Heart sound anomaly and quality detection using ensemble of neural networks without segmentation. In: Computing in Cardiology

29. Selesnick IW, Baraniuk RG, Kingsbury NG (2005) The dual-tree complex wavelet transform. IEEE Signal Process Mag 22:123-151. https://doi.org/10.1109/MSP.2005.1550194

30. Bianchi G (2007) Electronic Filter Simulation \& Design, 1st ed. McGraw-Hill Professional, NY

31. Al-Naami B, Owida H, Fraihat H (2020) Quantitative analysis signal-based approach using the dual tree complex wavelet transform for studying heart sound conditions. 2020 IEEE 5th Middle East Africa Conf Biomed Eng 1-4. https://doi.org/10.1109/MECBME47393.2020.9265121

32. Vermaak H, Philibert Nsengiyumva NL (2016) Using the Dual-Tree Complex Wavelet Transform for Improved Fabric Defect Detection. ournal Sens 2016:8. https://doi.org/https://doi.org/10.1155/2016/9794723

33. Daubechies I (1988) Orthonormal bases of compactly supported wavelets. Commun Pure Appl Math 41:. https://doi.org/10.1002/cpa.3160410705

34. Goodfellow J, Escalona OJ, Kodoth V, Manoharan G (2015) Efficacy of dwt denoising in the removal of power line interference and the effect on morphological distortion of underlying atrial fibrillatory waves in AF-ECG. In: 
IFMBE Proceedings

35. van Drongelen W (2007) Signal Averaging. In: Signal Processing for Neuroscientists. Elsevier, pp 55-70

36. Jang JSR (1993) ANFIS: Adaptive-Network-Based Fuzzy Inference System. IEEE Trans Syst Man Cybern 23:. https://doi.org/10.1109/21.256541

37. Goda MA, Hajas P (2016) Morphological determination of pathological PCG signals by time and frequency domain analysis. In: Computing in Cardiology. IEEE Computer Society, pp 1133-1136

38. Langley P, Murray A (2016) Abnormal heart sounds detected from short duration unsegmented phonocardiograms by wavelet entropy. In: Computing in Cardiology

39. Homsi MN, Medina N, Hernandez M, et al (2016) Automatic heart sound recording classification using a nested set of ensemble algorithms. In: Computing in Cardiology

40. Singh-Miller NE, Singh-Miller N (2016) Using spectral acoustic features to identify abnormal heart sounds. In: Computing in Cardiology. IEEE Computer Society, pp 557-560 